\title{
Interprofessional Collaboration between Physical Therapist and Nursing Students Using an Acute-Care Simulation: A Pilot Study
}

\section{Andrew Bartlett ${ }^{1}$ and Lynda J Dimitroff ${ }^{* 2}$}

${ }^{1}$ Department of Physical Therapy, Nazareth College of Rochester, USA

${ }^{2}$ Educational and Leadership Consultant, USA

*Corresponding author: Lynda J Dimitroff, Educational and Leadership Consultant, 66 Hinsdale Street, Rochester, 14620, NY, United States, Tel: 5852177886; E-mail: lynda.dimitroff@gmail.com

Received date: December 3, 2017; Accepted date: December 13, 2017; Published date: December 22, 2017

Copyright: ( 2018 Bartlett A, et al. This is an open-access article distributed under the terms of the Creative Commons Attribution License, which permits unrestricted use, distribution, and reproduction in any medium, provided the original author and source are credited

\begin{abstract}
Aim: The primary aim of the study was to describe an interprofessional experience between physical therapist and nursing students involving an acute-care laboratory setting using simulated patients. A secondary aim was to present findings on student's level of confidence in treating patients in an acute care setting.

Methods: A convenience sample $(\mathrm{N}=51)$ was used for this within groups study to explore self-reported confidence and perceived benefits/challenges among physical therapist and nursing students at baseline and following a 90-minute acute-care simulated laboratory experience. Debriefing sessions took place immediately after the simulation. Students were provided a survey via Survey Monkey one-week prior to and within one-week postacute-care simulation. Questions were slightly modified from previously used scales assessing student confidence in simulated experiences and working interprofessionally.
\end{abstract}

Results: A Wilcoxon signed-rank test determined that on average the students significantly improved their level of confidence from pre-test to post-test with all $p$ values $<0.05$, ranging from $p<0.001$ to 0.019 . Statistical analysis determined that students statistically improved their perception of working interprofessionally following an acute-care simulation.

Conclusion: Interprofessional education that consists of active learning between professions is vital to improve quality of patient care. The results of our pilot study suggest that an acute-care simulation between physical therapists and nursing students demonstrated improved confidence in clinical skills and a better understanding of interprofessional care.

Keywords: Acute-care; Interprofessional care; Interprofessional education; Nursing; Physical therapy; Simulation

\section{Introduction}

Interprofessional education has been recommended by the Institute of Medicine to improve collaboration among health care professionals and to ultimately improve quality of care to patients [1]. Interprofessional activities within an educational setting have been shown to improve attitudes and beliefs in working on teams [2]. Furthermore, interprofessional experiences provide students with opportunities to improve clinical competency and clinical decisionmaking skills within a low stakes environment; while at the same time allowing faculty to assess these skills [3]. Recently, an interprofessional experience between physical therapist (PT) and nursing students within an acute-care setting using a high-fidelity manikin (Sim Man) demonstrated increased feelings of competency and autonomy among physical therapy students. Additionally, PT students reported a better understanding of working on an interprofessional team and the importance of interprofessional collaboration within an acute-care environment [4]. To the best of our knowledge, there is a gap in the literature investigating the confidence and perception of interprofessional care in both physical therapy and nursing students within a simulated acute-care setting where students role play being a patient. Specific aims of this article were to:

- Describe an interprofessional experience between physical therapist students/faculty and nursing students/faculty within an acute-care simulation;

- Present preliminary findings on student's level of confidence in treating patients within an acute-care setting; and

- Present preliminary findings on student's perception in working within an interprofessional team in a simulated acute-care simulation.

\section{Methods}

\section{Study design}

A convenience sample was used for this within groups study to explore self-reported confidence and perceived benefits/challenges among physical therapist and nursing students at baseline and following a 90-minute acute-care simulated laboratory experience. 


\section{Setting and participants}

Data were collected from fifth year graduate physical therapist and third and fourth year nursing students within a liberal arts college located in Upstate New York. Fifty-one $(\mathrm{N}=51)$ individuals participated in this study including 45 physical therapist students and six nursing students. Participants were non-randomized into 12 different groups with each group comprising of approximately five participants (nursing students were assigned to more than one group). Participants were included if they were enrolled in either the Cardiovascular and Pulmonary Management course (physical therapist students), the Foundations of Transcultural Nursing, or Care of Adult course (nursing students). Two separate 90-minute sessions took place on the same day with each session having different participants with the exception of a few nursing students who participated in both sessions. Each session took place within a nursing laboratory (lab), which replicated an acute-care setting. The nursing lab included hospital beds, IV poles, mediation carts, oxygen, wallmounted blood pressure cuffs and a nurse's station.

\section{Intervention (Acute-care simulation)}

Interprofessional groups: Approximately one week prior to the acute-care simulation each participant received written learning objectives and expectations. The learning objectives included but were not limited to the following: performing a physical examination, interpreting medical information, developing and implementing a treatment plan and working interprofessionally by demonstrating effective communication skills. Additionally, each participant was provided a description of all the cases and was specifically assigned to one of them. It was expected that each student would review all the cases in order to be actively involved in the debriefing session. Cases were complex in nature and included information pertaining to laboratory values, ECG results, spirometry, wound care and medications. Specific diagnoses for each case included: coronary artery bypass graft, fractures, pneumonectomy, concussion, cerebral vascular accident and coma. Patient ages ranged from pediatrics to older adults. Each group had approximately 45 minutes to complete the learning objectives. Immediately following the acute-care simulation, a 30minutes debriefing session took place. The debriefing session was facilitated by the authors of this report, and included guided questions for each interprofessional group to answer. Example of questions included: what did you learn from the other profession, what you would have done differently if you could do the session over again and what other information would have been beneficial to have pertaining to your patient.

Simulated patients: Six physical therapist students who were not included in the study acted as simulated patients. The simulated patients arrived one hour prior to lab in order to be properly positioned in the hospital bed as well as to have the necessary lines, tubes and bandages placed. All six simulated patients were provided specific scripts. Scripts contained questions to ask the team and responses to the role-play.

Interprofessional faculty: Four physical therapy and one nursing faculty member assisted in the acute-care simulated lab. Participants were informed that faculty were there to encourage collaboration, trouble-shoot questions and to assist simulated patients with set-up and instructions. Participants were also instructed that faculty were there to ask specific questions and that the goal of the experience was to create a low-stakes learning environment.
Debriefing session: Debriefing sessions took place within the acutecare lab and immediately after the simulation. All faculty assisted with debriefing questions and each interprofessional team reflected on their performance and what they would do differently next time. Affirmations and suggestions were also provided by faculty members on what they observed that went well, and on how to improve interprofessional collaboration and clinical performance in the future.

\section{Measures}

The Human Subjects Research Committee at Nazareth College approved the study. Students were provided a survey via Survey Monkey one week prior to and within one week post-acute care simulation. Questions were slightly modified from previously used scales assessing student confidence in simulated experiences and working interprofessionally [5,6]. A total of 29 questions consisted of three main categories: confidence (7), interprofessional behavior (14) and other (8). In the area of confidence, students rated themselves using a 5-point Likert-type scale ranging from "1" representing "not confident" and "5" signifying "very confident". Confidence was assessed in making clinical decisions when the patient's physiological status changed, identifying lines/tubes, chart review, vital signs assessment, and physical examination. Students assessed their interprofessional behavior using a 6-point Likert-type scale in the areas of respect, understanding and cooperation. The scale ranged from a "1" representing "strongly disagree" to a " 5 " symbolizing "strongly agree". The "other" category also used a 6-point Likert-type scale and included questions regarding students' interest in working in acutecare, whether this simulation would be beneficial, and assessing their own profession (i.e., individuals in my profession are well-trained). Numbers on the scale ranged from a " 1 " indicating "not beneficial" to a " 5 " being "very beneficial". In addition to the quantitative questions, participants were provided an opportunity to write comments.

\section{Statistical analysis}

A Wilcoxon signed-rank test comparing median differences between the same participants from baseline to post-testing was used for each question. Data were analyzed using IBM SPSS statistics (version 23). A statistical significance level was set at $\mathrm{p}<0.05$.

\section{Results}

A total of 51 participants were used in the analysis to assess students' confidence in working within an acute-care-setting. Furthermore, students' perception assessed working on an interprofessional team. Table 1 displays the median scores of participant's perception of confidence pre and post using the Likerttype scale. A Wilcoxon signed-rank test determined that on average the students significantly improved their level of confidence from pre to post with all $\mathrm{p}$ values $<0.05$, ranging from $\mathrm{p}<0.001$ to 0.019 . Table 2 displays the median scores of the questions pertaining to participant's interprofessional behavior. A majority of the questions demonstrated a statistical improvement from pre to post in participant's perception of working interprofessionally. In the "other" category six out of the eight questions were not statistically significant, $p>0.05$. Figures 1 and 2 demonstrate percent change for examples of acute-care confidence and interprofessional collaboration. Likert-type scales were combined to allow data to be more easily viewed. 
Citation: Bartlett A, Dimitroff LJ (2018) Interprofessional Collaboration between Physical Therapist and Nursing Students Using an Acute-Care Simulation: A Pilot Study. J Nurs Care 7: 443. doi:10.4172/2167-1168.1000443

Page 3 of 4

\begin{tabular}{|l|l|l|l|l|l|}
\hline Question \# & Description (Confidence) & Median Pre & Median Post & Difference & Significance (2-tailed) \\
\hline 19 & Evaluate and treat safely & 2 & 3 & -1 & 0 \\
\hline 20 & Identifying equipment & 2 & 4 & -1 & 0 \\
\hline 21 & Making clinical decisions & 3 & 4 & -1 & 0 \\
\hline 22 & Responding to changes & 3 & 4 & -1 & 0 \\
\hline 26 & Interpreting changes & 4 & 4 & 0 & -1 \\
\hline 27 & Chart review & 3 & 4 & 0 & 0 \\
\hline 28 & Vital sign assessment & 4 & 4 & 0.019 \\
\hline
\end{tabular}

Table 1: Median scores for participant's confidence pertaining to acute care.

\begin{tabular}{|c|c|c|c|c|c|}
\hline Question \# & Description & Pre & Post & Difference & Significance (2-tailed) \\
\hline 2 & Work closely with other professions & 5 & 6 & 0 & 0.016 \\
\hline 4 & Respect work by my profession & 5 & 5 & 0 & 0.085 \\
\hline 6 & Need to cooperate with other professions & 6 & 6 & 0 & 0.791 \\
\hline 8 & Depend on work of other professions & 5 & 5 & 0 & 0.012 \\
\hline 9 & Others think highly of my profession & 5 & 5 & 0 & 0.051 \\
\hline 11 & Individuals feeling of higher status & 6 & 6 & 0 & 0.821 \\
\hline 14 & Willingness to share information & 6 & 6 & 0 & 0.016 \\
\hline 15 & Have good relations with other professions & 5 & 5 & 0 & 0.012 \\
\hline 16 & Think highly of others & 5 & 5 & 0 & 0.009 \\
\hline 17 & Work well with others & 5 & 6 & 0 & 0.049 \\
\hline 18 & Seek advice of others & 5 & 5 & 0 & 0.043 \\
\hline 24 & Confidence in working with other professions & 4 & 4 & 0 & 0 \\
\hline 29 & $\begin{array}{l}\text { Confidence in working with others for an educational } \\
\text { experience }\end{array}$ & 4 & 4 & 0 & 0.019 \\
\hline
\end{tabular}

Table 2: Questions pertaining to working interprofessionally.

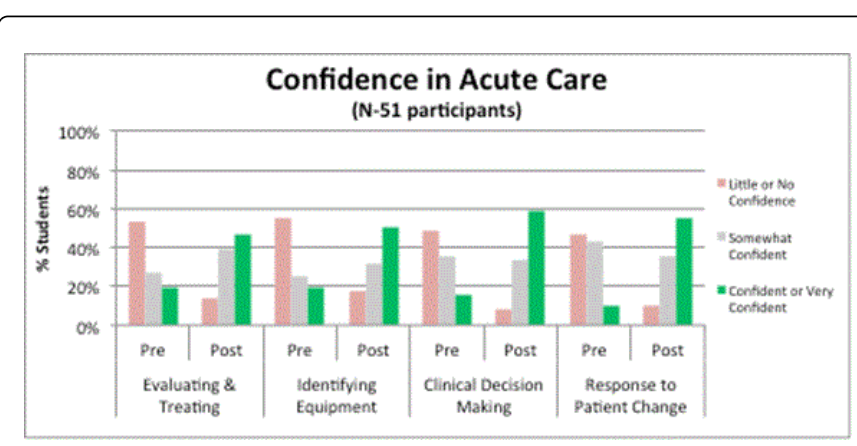

Figure 1: Examples of four questions pertaining to acute-care confidence from pre to post simulated experience.

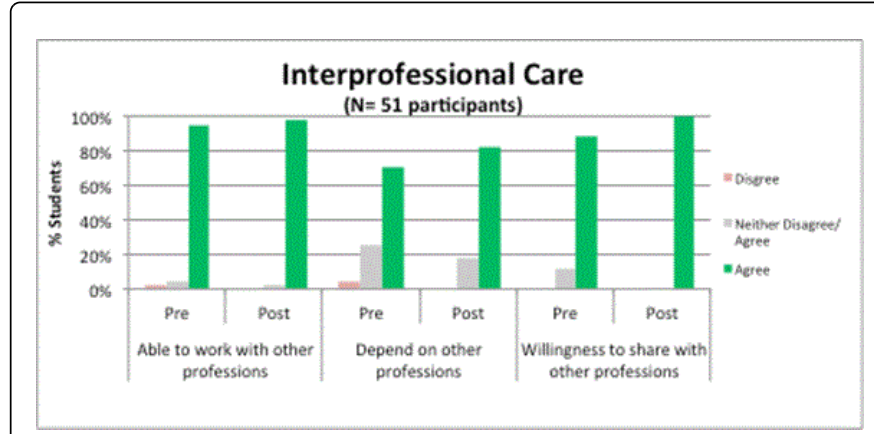

Figure 2: Examples of four questions pertaining to perception of interprofessional care from pre to post simulated experience. 


\section{Discussion}

Interprofessional education and collaboration are recommended by the Institute of Medicine [1]. Despite this recommendation, the body of literature describing the effectiveness of an interprofessional experience between physical therapist and nursing students is limited [4]. We investigated the effectiveness on an acute-care simulation among physical therapist and nursing students working interprofessionally. To the best of our knowledge, this is the first report to describe this type of experience. Results clearly demonstrated that this type of interprofessional experience was beneficial to improve confidence in the acute-care setting. Furthermore, this simulation improved students' perception of interprofessional collaboration. Two questions relating to interprofessional care were not statistically significant. The participants rated themselves relatively the same from pre to post survey. Additionally, participants assessed themselves as "moderately agree" or "strongly agree" indicating they viewed interprofessional collaboration in a positive nature. The same explanation can be made for questions in the "other" category. For example the question "how beneficial will exposure to a simulated patient with multiple lines/tubes be for you in terms of an educational experience"; $38 \%$ reported it being beneficial and $60 \%$ stated very beneficial at baseline. At post-testing the percentages stayed approximately the same.

\section{Strengths and Limitations}

Through our investigation, we identified several areas that made this interprofessional acute-care simulation successful: (1) there was collaboration among nursing and physical therapy faculty in planning and during the activity, (2) both physical therapist and nursing students were actively engaged throughout, (3) the location of the experience took place in a simulated hospital setting that included hospital beds and all the medical equipment needed to use with our simulated patients, (4) we emphasized a low-stakes fun environment that facilitated collaboration and learning. Although a formal qualitative analysis was not conducted participants who wrote comments spoke favorably about the interprofessional experience. Two quotes included:

- "Being able to work with the nurses helps us as PTs see the patient from a different perspective and look for things we normally wouldn't." (PT student)

- "This experience was helpful because both the PT students and I were able to learn about the others profession and how we benefit each other when it comes to caring for the patient." (Nursing student)

One limitation of the present study was the decision to use a few nursing students to participate in both 90 -minute sessions. This may have influenced the nursing student's responses, due to being exposed to the experience more than the physical therapist students. This choice to use the same nursing students in both sessions was made based on not having enough nursing students to assist with the second simulation session. Future studies should include an equal number of nursing and physical therapist students in both sessions to ensure equal exposure. The most important limitation of the present review was selecting and using only 12 questions from the Interdisciplinary Education Perception Scale. The Interdisciplinary Education Perception Scale consists of 18 questions and has been shown to have good validity [6]. We chose a limited number of questions from this scale to cut down on the total number of questions and length of time to complete the survey. Ideally, the complete Interdisciplinary Education Perception Scale should have been used to limit any question of reliability and validity of the modified scale that was used.

\section{Conclusion}

Interprofessional education that consists of active learning between professions is vital to improve quality of patient care. The results of our study suggest that an acute-care simulation between physical therapists and nursing students demonstrated improved confidence in clinical skills and a better understanding of interprofessional care. Future studies should use the full Interdisciplinary Education Perception Scale as well as a validated and reliable outcome measures to assess clinical competence within an acute-care setting. Furthermore, futures studies should include other professions i.e., creative art therapy, occupational therapy, and social work and more robust qualitative analysis of written comments and/or focus groups.

\section{Acknowledgement}

The authors thank all the participants and the faculty members who volunteered their time to assist in the laboratory experience - Karen Hertline, JJ Mowder-Tinney and Mary Ellen Vore.

\section{References}

1. Institute of Medicine (2015) Measuring the impact of interprofessional education on collaborative practice and patient outcomes. Committee on measuring the impact of interprofessional education on collaborative practice and patient outcomes. Board on Global Health, National Academy of Sciences, Washington DC.

2. Brock D, Abu-Rish E, Chiu C, Hammer D, Wilson S, et al. (2013) Interprofessional education in team communication: Working together to improve patient safety. Br Med J Qual Saf 22: 414-423.

3. Silberman NJ, Panzarella KJ, Melzer BA (2013) Using human simulation to prepare physical therapy students for acute care clinical practice. J Allied Health 42: 25-32.

4. Lefebvre K, Wellmon R, Ferry D (2015) Changes in attitude towards interprofessional learning and collaboration among physical therapy students following a patient code simulation scenario. Cardiopulm Phys Ther J 26: 8-14.

5. Bednarek M, Downey P, Williamson A (2014) The use of human simulation to teach acute care skills in a cardiopulmonary course: A case study. J Phys Ther Educ 28: 27-34.

6. Luecht RM, Madsen MK, Taughter MP, Petterson BJ (1990) Assessing professional perceptions: Design and validation of an interdisciplinary education perception scale. J Allied Health 19: 181-191. 